\title{
ARGO ALS KRIEGSSCHIFF UND ALS FRACHTSCHIFF: ASTRALIKONOGRAPHIE ZWISCHEN ANTIKE UND MITTELALTER
}

\section{ZUSAMMENFASSUNG}

Die 48 Konstellationen des antiken Sternenhimmels hat man im Altertum als Figuraldarstellungen mit Menschen, Tieren oder Sachen dargestellt. Die Griechen und Römer folgten dabei den Vorbildern aus Mesopotamien und Ägypten, sie formten jedoch die Bilder der Sternkonstellationen auf eigene Weise. Das entscheidende Medium war die Buchmalerei. In der Wahl der Motive spiegelt sich das Leben und die Erfahrungswelt der Illustratoren. So hat man für das Sternbild der Gemini (Zwillinge) im Tierkreis zwei verschiedene Mythenbilder ausgewählt. Für das weit im Süden gelegene Sternbild des Schiffes (Argo) griff man auf aktuelle Schiffsbilder zurück, in einem Falle sogar auf einen ganz modernen Kriegsschifftypus der römischen Marine. Die mittelalterlichen Buchausgaben der antiken Astronomen halten sich bei den Illustrationen noch in vielen Punkten an die antiken Vorbilder.

KEY WORDS: STERNENHIMMEL, IKONOGRAPHIE, ANTIKE, MITTELALTER.

\section{ANTIKE UND MITTELALTERLICHE BILDQUELLEN}

Für die Ikonographie antiker Sternbilder stehen uns im Bereich des Tierkreises (Zodiakos, Zodiacus) unzählige Bildwerke aus dem Altertum zur Verfügung ${ }^{1}$. Dies betrifft aber nur die zwölf Tierkreiszeichen; es bleiben die übrigen 36 Sternbilder des antiken Sternenhimmels, dessen Konstellationen die Alten auf 48 begrenzten ${ }^{2}$. Hier sind

1 Gundel 1992.

2 Thiele 1898; Schlachter und Gisinger 1927; Gundel 1992; Künzl 2000; idem 2005.- Für die moderne Zeit hat die Internationale Astronomische Union (IAU) im Jahre 1922 eine Liste von 88 verbindlich festgelegten Sternbil- die antiken Bildquellen schon spärlicher. Ganz erhalten präsentiert sich der Mainzer Himmelsglobus (Abb. 1); am Globus des Atlas Farnese fehlen einige Bilder (Abb. 2). Auch die Planisphäre der Salzburger Kalenderuhr ist leider nur ein kleines Fragment; ihre Bilder wären sonst eine entscheidende Hilfe gewesen.

Umso dankenswerter ist es, dass eine Reihe mittelalterlicher Himmelskarten (Planisphären) die antiken Bildtraditionen fortsetzt. Planisphären sind flache Sternkarten, mit dem Nordpol im Zentrum, dem exzentrischen Ekliptikpol und dem ebenfalls exzentrischen Ring der Ekliptik; sie

dern erstellt. 


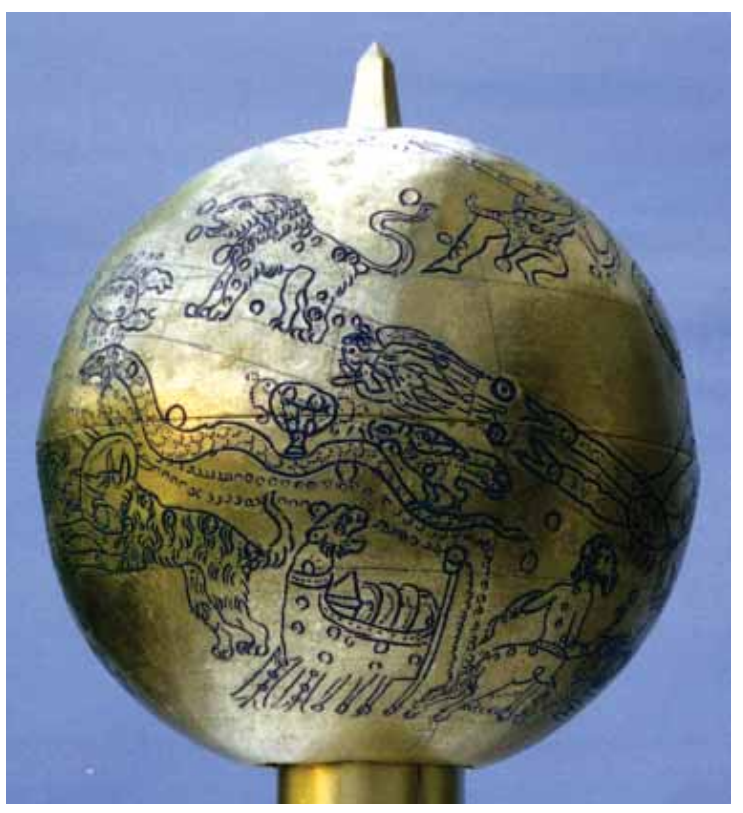

Abb. 1

Römischer Himmelsglobus. Messing. Dm. 110 mm. 150-220 n. Chr. Mainz, Römisch-

Germanisches Zentralmuseum Inv. O.41339.

Galvanoplastische Kopie mit dunkler Einfärbung der Linien. Fot. Verf.

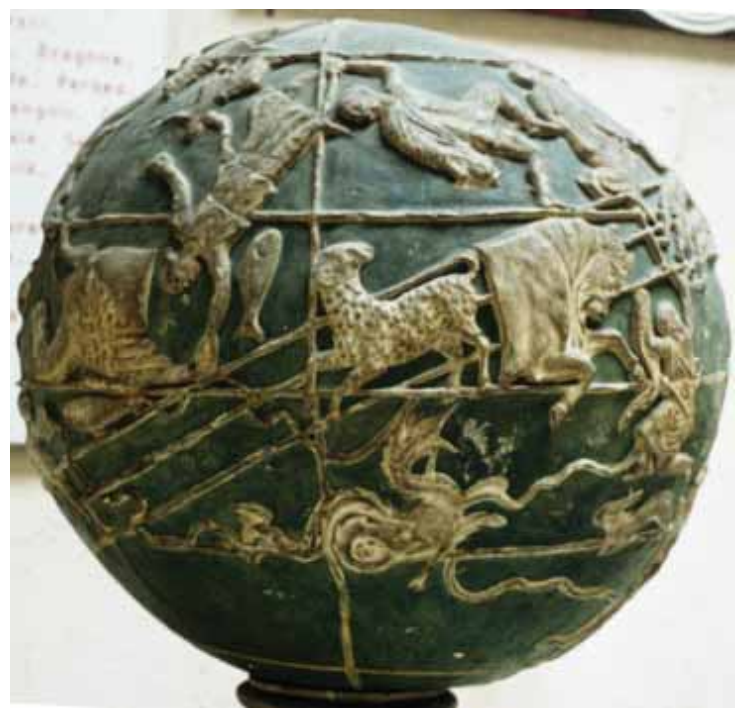

Abb. 2

Römischer Himmelsglobus. Globus des Atlas Farnese. Marmor. Dm. 65 cm. Um Christi Geburt. Neapel, Nationalmuseum Inv. 6374. Kopie im Museo della Civiltà Romana, Rom. Fot. Verf. können entweder nur in der Form der Hemisphäre die nördlichen Sternbilder bieten, oder man nimmt bis zum südlichen Wendekreis die dann nach außen immer mehr auseinanderliegenden Sternbilder auf.

Der Wunsch nach flachen Sternkarten muß bereits im Hellenismus oder der frühen Kaiserzeit aufgekommen sein. Außerdem konnte man Planisphären in die Bücher als Illustration aufnehmen. Im 2. Jh. n. Chr. existierten solche Sternkarten sicherlich, denn ihre Konstruktion wird von Claudius Ptolemaeus beschrieben. Außerdem haben wir ungefähr aus derselben Zeit das einzige antike Fragment einer Planisphäre, die Salzburger Kalenderuhr (Abb. 3). Im Mittelalter sind die farbigen Planisphären der Prachtausgaben der antiken Astronomieautoren (Aratos, Ptolemaeus u.a.) unsere Hauptquelle ${ }^{3}$.

\section{BESONDERHEITEN DER ANTIKEN IKONOGRAPHIE}

Eine umfassende ikonographische Analyse der antiken und mittelalterlichen Sternbildfassungen ist noch nicht geschrieben worden. Lohnen würde sie sich schon. So konnte man feststellen, dass das Sternbild Widder (Aries) meist als aufrecht gehendes oder springendes Tier dargestellt wird (z. B. auf dem Globus Farnese; Abb. 2) ${ }^{4}$. Wenn er einen Reif trägt oder durch einen Reif zu springen scheint, wie im Codex Vossianus lat. 79 Fol. $34 v^{5}$, so ist damit der Äquinoktialkolur gemeint. Den ruhenden Widder, so wie wir ihn auf dem Mainzer Globus sehen (Abb. 4), finden wir auf einer kleinen Reihe von Belegen, die alle ins hellenistische und römische Ägypten weisen. Die Planisphäre im Codex Vaticanus graec. 1087 (fol. 310v) aus dem 15. Jahrhundert zeigt neben dem ruhenden Widder einen anderen Hinweis auf

3 Saxl 1915a; idem 1915b; Saxl u.a. 1953; von Euw 1987. 4 Thiele 1898: 27 Abb. 5 u. Taf. 4 oben.

5 Thiele 1898: 108 Fig. 33; Gundel 1992: 328 Nr. 460 (2) Abb. S. 329. 
Ägypten: Ara (Altar) im Süden ist in dieser Handschrift leuchtturmartig nach Art des Pharos von Alexandrien wiedergegeben.

\section{DIE GEMINI, BEISPIEL FÜR EINE IKONOGRAPHISCHE PARALLEL- TRADITION}

Die Gemini (Zwillinge) erscheinen auf dem Mainzer Globus als zwei nackte Jünglinge, die sich umarmen (Abb. 5); die Gruppe entspricht damit jenen antiken Auffassungen der Gemini, welche sie als Castor und Pollux, die Dioskuren (Söhne des Zeus-Iuppiter) sahen. Die Darstellung der beiden nackten Jünglinge, die sich hier an den Dioskuren (freilich ohne ihre Piloi, die Filzkappen der Seeleute) orientiert, konnte im Altertum verschiedenen mythischen Gestalten angeglichen werden (Amphion und Zethos, die Kabiren von Samothrake).

Auf der Salzburger Kalenderuhrscheibe (Abb. 3) ist der eine noch erhaltene Zwilling als Hercules aufgefaßt, man muß also den zweiten als Apollo ergänzen 6 . Im Tempelschatz von Marengo (Alessandria/Italien) erscheinen die Gemini ebenfalls als Hercules mit der Keule und Apollo mit der Leier (Abb. 6) ${ }^{7}$. Die Gemini als Hercules und Apollo sind durch alexandrinische Münzen der Zeit des Antoninus Pius bezeugt, was man mit dem astrologischen Tetrabiblos des Ptolemaeus in Verbindung brachte ${ }^{8}$. Auch auf der Tabula Bianchini im Louvre finden sich die Gemini als Hercules mit der Keule und Apollo mit der Kithara ${ }^{9}$. Dasselbe gilt für das Marmorrelief Daressy ${ }^{10}$. In

6 Maass 1902: 196; Künzl 2000: 511 Taf. 64,4.

7 Künzl 2000: 511 Taf. 63,1.

8 Thiele 1898: 67-69 Abb. 13; Lehr 1971: 8 Abb.- Vgl. auch die in den von F. Boll vorgelegten Texten vorherrschende Interpretation als Hercules und Apollo: Boll 1903: 122128.

9 Boll 1903: 299-305 Taf. 5; Gundel 1992: 110f. Abb. 51. 226 Nr. 63; Abry 1993: Taf. VI; Stückelberger 1994: 41.

10 Boll 1903: 305-306 Taf. 6; Neugebauer und Parker 1969: 103 Nr. 80 Taf. 40B; Gundel 1992: 226 Nr. 62. Abb.

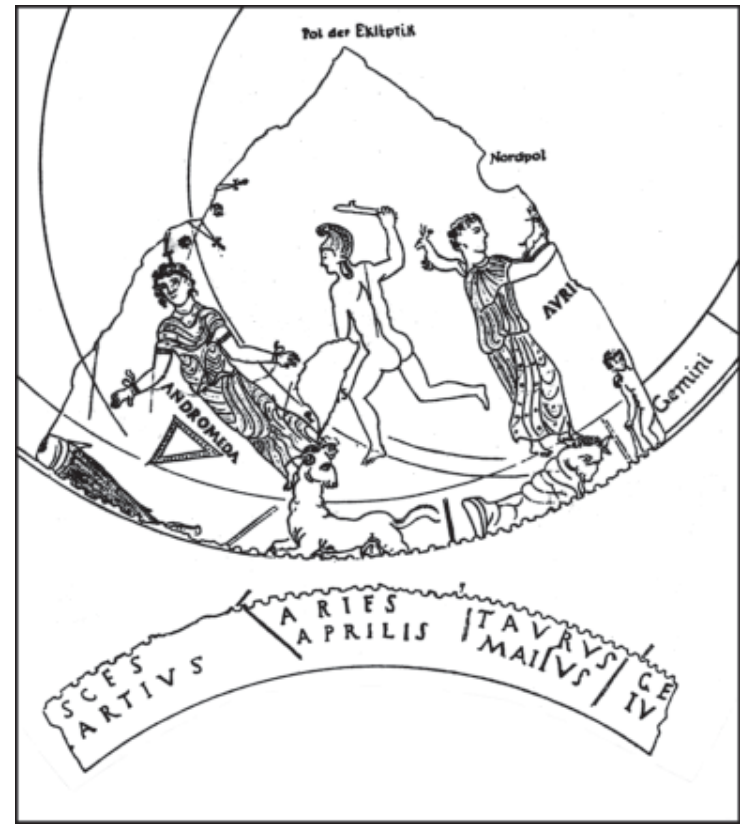

Abb. 3

Flache Sternenkarte (Planisphäre) als Teil einer Kalenderuhr. Bronze. Aus Salzburg/Österreich. Planisphärenfragment einer Kalenderuhr. Aus Salzburg/Österreich. Bronze. 2. Jh. n. Chr. Salzburg, Museum Carolino-Augusteum. Nach Benndorf u.a. 1903, 39 Fig. 18.

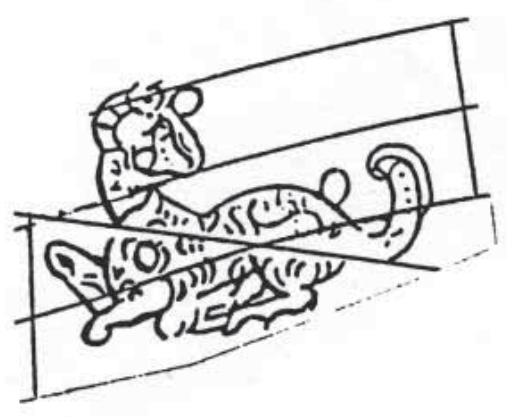

Abb. 4

Liegender Widder (Aries) vom Mainzer Himmelsglobus (vgl. Abb. 1).

150-220 n. Chr. Zeichnung Julia Ribbeck, Mainz, Römisch-Germanisches Zentralmuseum. 


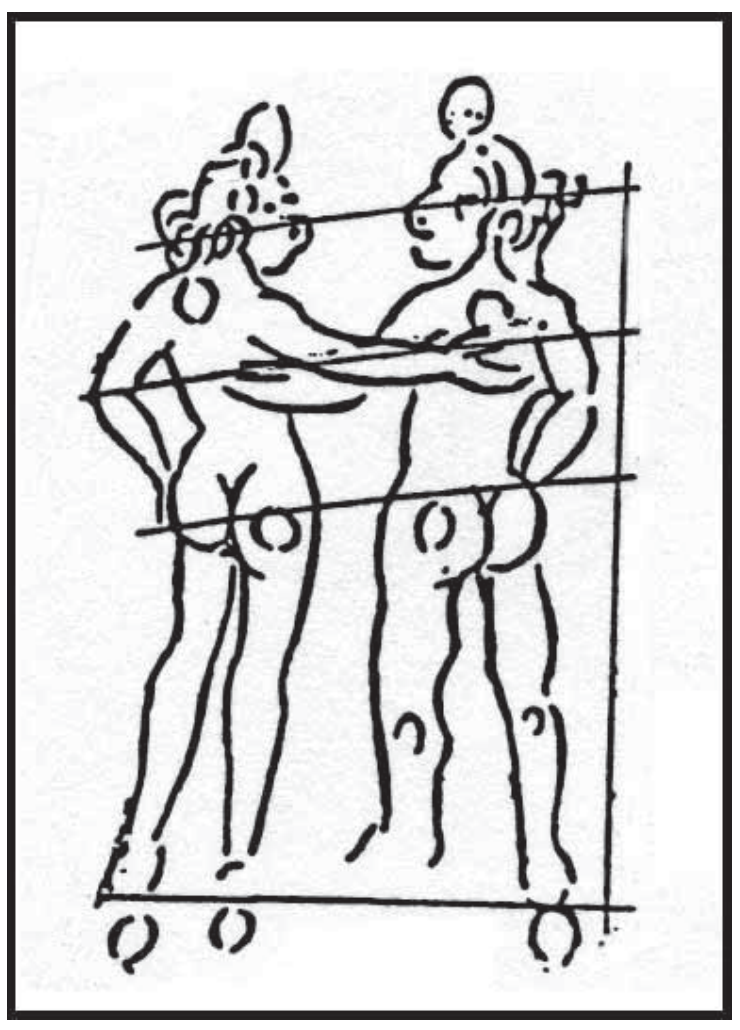

Abb. 5

Die Zwillinge (Gemini) vom Mainzer Himmelsglobus (vgl. Abb. 1).

150-220 n. Chr. Zeichnung Julia Ribbeck, Mainz, Römisch-Germanisches Zentralmuseum.

der Tat sind die genannten Zeugnisse alle in das 2. Jh. n. Chr. (Marengo, Salzburg, Münzen aus Alexandrien) oder ungefähr in diese Zeit (Tabula Bianchini, Relief Daressy) zu datieren, der vermutete Einfluß des Claudius Ptolemaeus ist deshalb nicht unwahrscheinlich. Nun sind auch Hercules und Apollo Söhne des Iuppiter, wenn auch keine Zwillinge wie die Ledasöhne Castor und Pollux (griech. Kastor und Polydeukes).

Auf den mittelalterlichen Planisphären sind die Gemini im Codex Vaticanus graec. 108711 vielleicht ebenfalls noch auf das Paar Apollo-Hercules zu beziehen; der linke stützt sich auf einen Rest einer Kithara, der andere auf eine Art dünnen

S. 227; Abry 1993: Taf. II,1.

11 Künzl 2000: 511 Taf. 65,2.

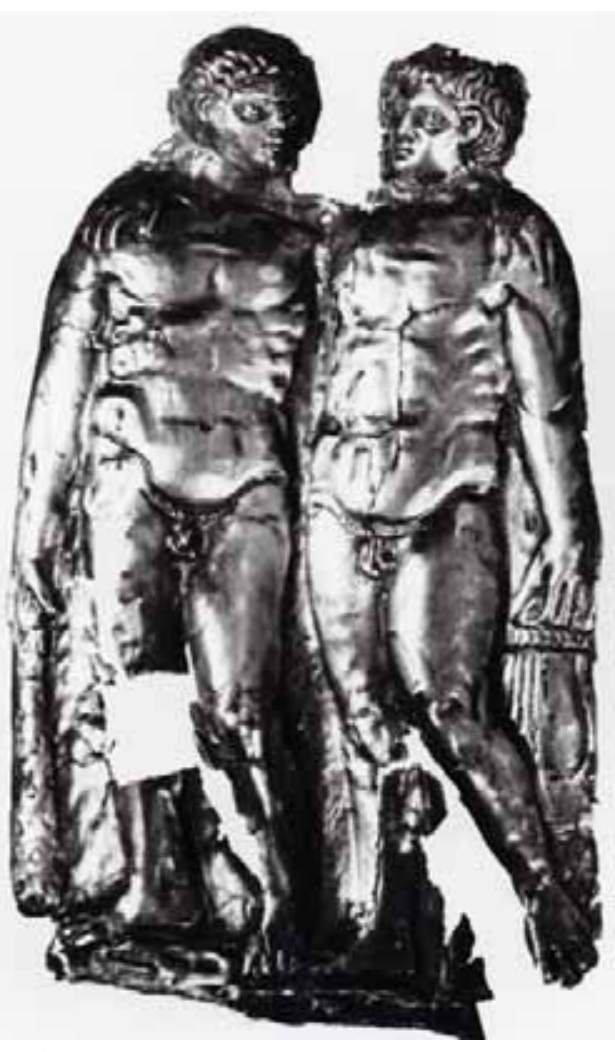

Abb. 6

Hercules und Apollo als Zwillinge (Gemini). Silberapplike aus einem Tempelschatz. 2. Jh. n. Chr. Aus Marengo, Alessandria/Italien. H. 17 cm. Turin, Museo di Antichità. Fot. Mainz, RömischGermanisches Zentralmuseum T 63/3296.

Stecken, in dem der Rest der Keule zu verstehen ist. Diese Deutung empfiehlt sich auch deswegen, weil die Darstellung des Zodiacus derselben Handschrift als Ring um Sonne und Mond die Gemini deutlich als Hercules und Apollo zeigt ${ }^{12}$. Deutlich ist die Keule am linken Zwilling des Codex Harleianus $647 \mathrm{zu}$ sehen, wobei in diesem Falle sein Gefährte ein Objekt hält, das auf den ersten Blick wie eine Lanze aussieht; da der Schaft der Waffe aber sehr kurz ist und auch unten keine Beschädigung erkennbar ist, muß es ein Pfeil sein, was wieder einen Apollo kennzeichnet. Die Gemini mit Keule (erkennbar, aber fast wie ein Hirtenstab geformt) des Codex Bernensis 88 gehen ebenso wie die Darstellung des Codex

$\overline{12 \text { Gundel 1992: } 319}$ Nr. 424. 


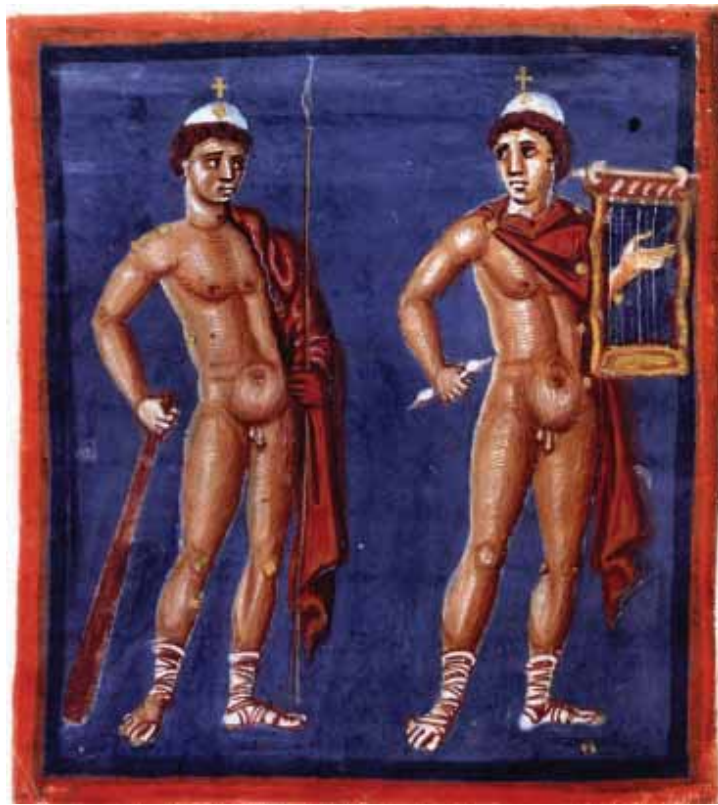

Abb. 7

Die Zwillinge (Gemini) als Mischung von Hercules und Apollo mit den Filzmützen der Dioskuren Castor und Pollux. Codex Vossianus lat. Q 79 fol. 16v. Um 840 n. Chr. Leiden, Universiteitsbibliotheek. Foto: Leiden, Universiteitsbibliotheek.

Bononiensis 188, auf dem ebenfalls die Keule erkennbar ist, die Kithara aber nicht (gleichfalls wie am Codex Bernensis 88) auf das Vorbild des Leidener Codex Vossianus lat. 79 zurück: Dort tragen die Gemini zwar die Filzkappe der Dioskuren $(\text { Abb. } 7)^{13}$, die freilich nun statt eines Sternes wie im Altertum ein Kreuz bekrönt. Zugleich aber hat der Künstler mit der Zither (Kithara) den einen als Apollo und mit der Keule den anderen als Hercules bezeichnet.

Die Hercules-Apollo-Tradition der Gemini, seit dem 2. Jh. n. Chr. nachweisbar und besonders mit Ägypten verbunden, erweist sich also als sehr langlebig.

13 Thiele 1898: 98 Fig. 24; Gundel 1992: 328 Nr. 460 (2) Abb. S. 329.

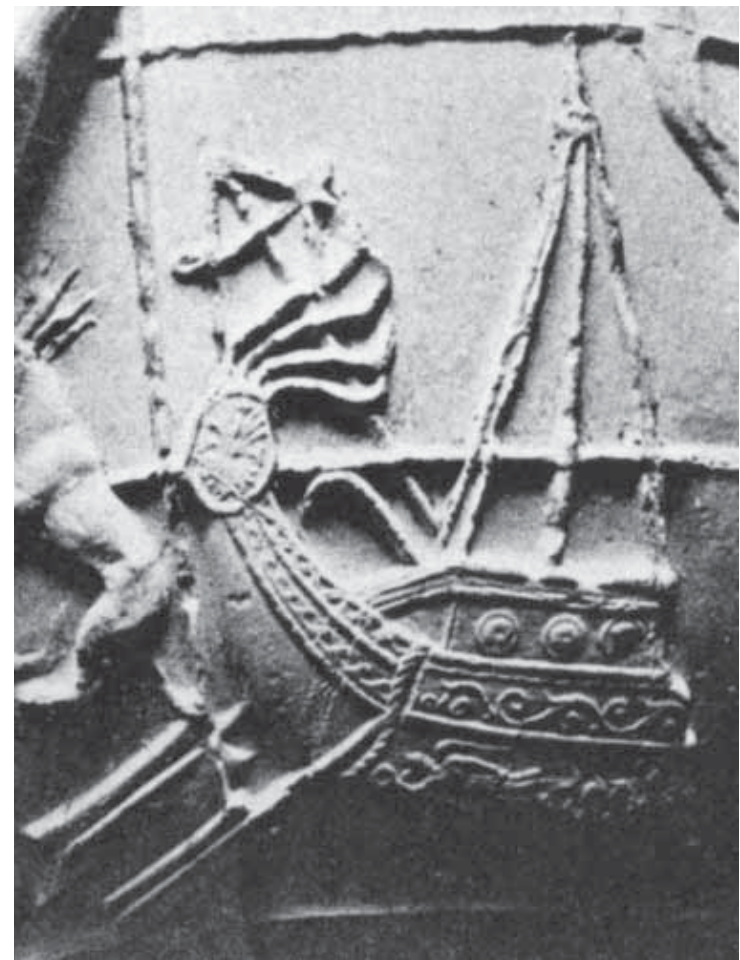

Abb. 8

Das Schiff (Argo) auf dem Globus des Atlas Farnese. Marmor. Um Christi Geburt. Neapel, Nationalmuseum Inv. 6374. Nach Thiele 1898, Taf. 5 oben.

\section{KRIEGSSCHIFFE UND FRACHTSCHIFFE}

Argo, das große Schiff am Himmel des Südens, wird als Halbschiff dargestellt. Hier fällt zunächst auf, dass es am Mainzer Globus (Abb. 1) ganz anders als auf dem Globus des Atlas Farnese (Abb. 2) gestaltet ist. Auf dem marmornen, großformatigen Himmelsglobus des Atlas Farnese (Abb. 8), den man sich bunt bemalt vorstellen muß, erscheint ein frühkaiserzeitliches Schiff mit Mast, zwei Steuerrudern und großer Heckzier (Aphlaston): Dargestellt ist die hintere Hälfte des Schiffes ${ }^{14}$. Die Riemenreihen für eine Rudererbesatzung fehlen, also dürfte ein Frachtschiff gemeint sein. Wie wenig der entwerfende Meister Genauigkeit anstrebte, zeigt sich in dem

14 Thiele 1898: Taf. 5 oben. 


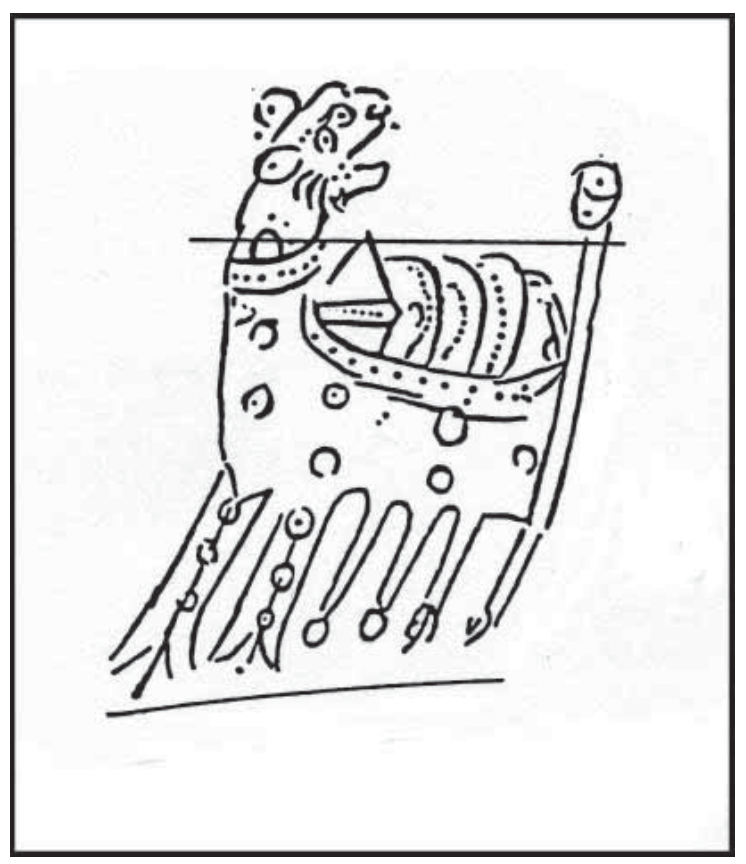

Abb. 9

Das Schiff (Argo) als Kriegsschiff am Mainzer Himmelsglobus (vgl. Abb. 1).

150-220 n. Chr. Zeichnung Julia Ribbeck, Mainz, Römisch-Germanisches Zentralmuseum.

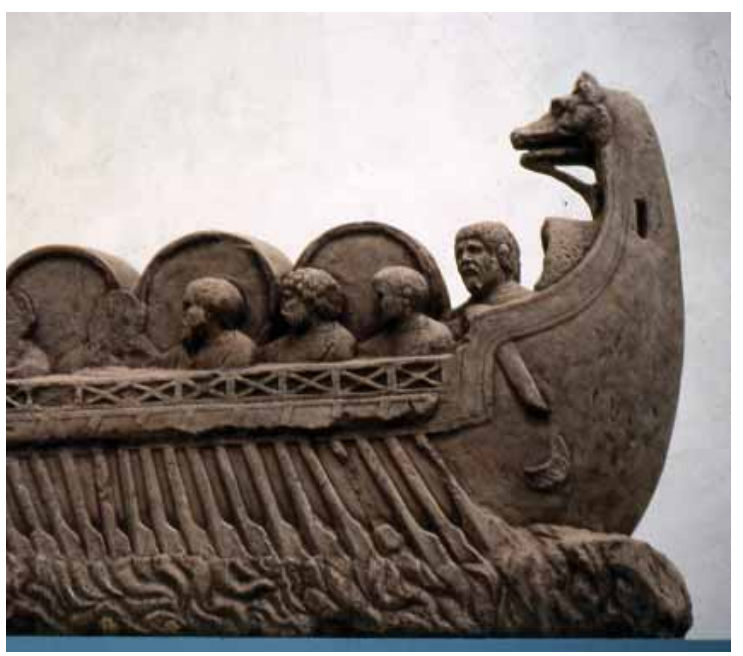

Abb. 10

Das Neumagenschiff. Teil eines Grabmals von Trier, gefunden in Neumagen an der Mosel. Um 200 n. Chr. Gerudertes Kriegsschiff, als Weintransportschiff außerplanmäßig eingesetzt. Trier, Rheinisches Landesmuseum. Kopie Mainz, Museum für Antike Schiffahrt. Fot. Verf.
Detail der runden Schilde als Dekor der oberen Bordwand; dies ist ein Motiv, welches man von der Darstellung römischer Kriegsschiffe kennt, beispielsweise von einem Relief aus Praeneste (Palestrina, in der Nähe von Rom) ${ }^{15}$.

Auch das Schiff Argo des Mainzer Himmelsglobus erscheint als Halbschiff, hier allerdings mit einem Tierkopf am Heck, welcher über das Deck nach vorne schauen soll (Abb. 9) ${ }^{16}$. Das Schiff ist ein Ruderschiff mit zwei breiten Steuerrudern hinten. Auf dem Deck steht direkt neben dem Heck eine Art Haus mit Giebel, worunter eine Kajüte zu verstehen ist, während die gebogenen Objekte wohl Schilde darstellen sollen.

Diesen militärischen Ruderschifftypus kennt man bisher aus dem Norden des Römerreiches, beispielsweise aus Darstellungen wie dem Weinschiff von einem Grabdenkmal des frühen 3. Jhs. n. Chr. aus Neumagen, Rheinland-Pfalz im Landesmuseum Trier (Abb. 10) ${ }^{17}$. Auch auf der hundert Jahre früher geschaffenen Traianssäule in Rom sind solche schnellen, schlanken Militärboote auf der Donau zu sehen (Abb. 11) ${ }^{18}$. Es handelt sich um binnentaugliche Biremen des 2 . bis 4. Jahrhunderts, zweireihige Riemenfahrzeuge mit Einzelbemannung (Biremes) ${ }^{19}$, welche für die Organisation der Reichsverteidigung an den Nordgrenzen sehr wichtig waren. Die römischen Reichsgrenzen zwischen Nordsee und Schwarzem Meer waren überwiegend Flussgrenzen, welche mit Hilfe von Binnenflotten verteidigt werden mussten.

Der Mainzer Himmelsglobus (Abb. 1) ist vermutlich im römischen Ägypten entworfen worden; seinem Künstler lag für das Schiff Argo ein Entwurf vor, der ein modernes, schnelles und nicht sehr großes Militärboot darstellte. Das heißt freilich nicht, dass nicht auch vor dem Mainzer

15 Bockius 2007: 45 Abb. 48.

16 Künzl 2000: 521 Abb. 13 Nr. 33.

17 Bockius 2007: 57 Abb. 59.

18 ibidem: 65 Abb. 68.

19 ibidem: 53-58.- Allgemein zur antiken Seefahrt vgl. auch Bockius 2006; Casson 1994; Höckmann 1985. 
Globus (150-220 n. Chr.) dieser Schiffstypus einmal in der Sternbildikonographie zitiert worden war. Hier fehlen uns bisher Belege aus der entscheidend wichtigen Buchillustration ${ }^{20}$. Die uns vorliegenden außerordentlich seltenen antiken Himmelsgloben wie jene in Mainz (Abb. 1) und in Neapel (Abb. 2) sind außerdem keine professionellen Astralgloben antiker Astronomen, sondern Dekorationsstücke, wobei freilich kein Zweifel besteht, dass sich die Künstler dabei an die Ikonographie der astronomischen Fachliteratur gehalten haben.

Von den mittelalterlichen Bildern des Schiffes Argo überliefern die einen in mehr oder weniger großer Vereinfachung den frühkaiserzeitlichen Typ des Atlas Farnese, also ein Schiff ohne Riemenreihe und mit einer Heckzier. Man findet diesen Frachtschifftypus in der Planisaphäre des Codex Bernensis 88 (Abb. 13) ${ }^{21}$, welcher vor $1029 \mathrm{zu}$ datieren ist. Sehr ähnlich und auf die gleiche Bildquelle zurückgehend erscheint das Schiff im Codex Bononiensis 188 (Planisphäre, fol. 20r. $)^{22}$, welcher in das 10.-11. Jahrhundert gehört. In beiden Fällen fehlt das Steuerruder. Dieses wiederum ist am Frachtschiff der Planisphäre des Codex Harleianus 647 aus dem 9. Jahrhundert angegeben (Abb. 12) 23. $^{23}$

Daneben hält sich über das gesamte Mittelalter die andere Tradition der Argodarstellung in Form eines Kriegsschiffes. Der Codex Vaticanus graec. 1087 (Abb. 15) ${ }^{24}$, der schon in die zeit der Renaissance des 15. Jahrhunderts gehört, weist sowohl die Ruderreihe wie auch die Kajüte auf dem Deck auf.

Daß diese Version nicht allein steht, zeigt das Ruderschiff dieses Typs des Hemisphärenbildes im Codex Sangallensis 250 aus dem 9. Jahr-

20 Stückelberger 1994.

21 Künzl 2000: Farbtafel IX,2.

22 ibidem: Farbtafel IX,1.

23 ibidem: Taf. 66.

24 Boll 1903: 92 Taf. 1; Gundel 1992: 312 Nr. 396. Abb. S. 311; Stückelberger 1994: 41 Abb. 19; Künzl 2000: Taf. 65,2 .

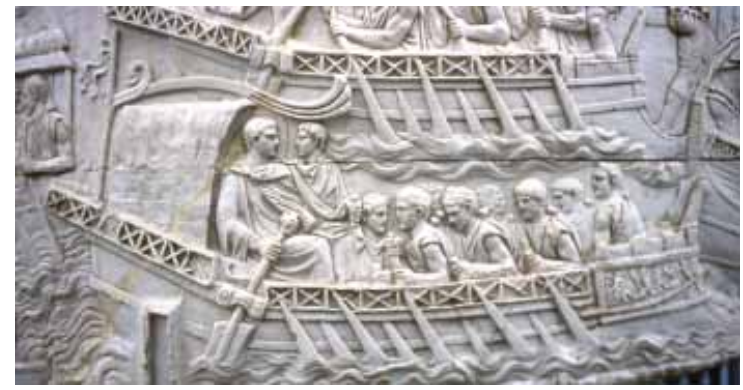

Abb. 11

Rom, Traianssäule. Konvoi leichter Militärboote auf der Donau. Frühes 2. Jh. Kopie im Museum für Antike Schiffahrt, Mainz. Fot. Verf.

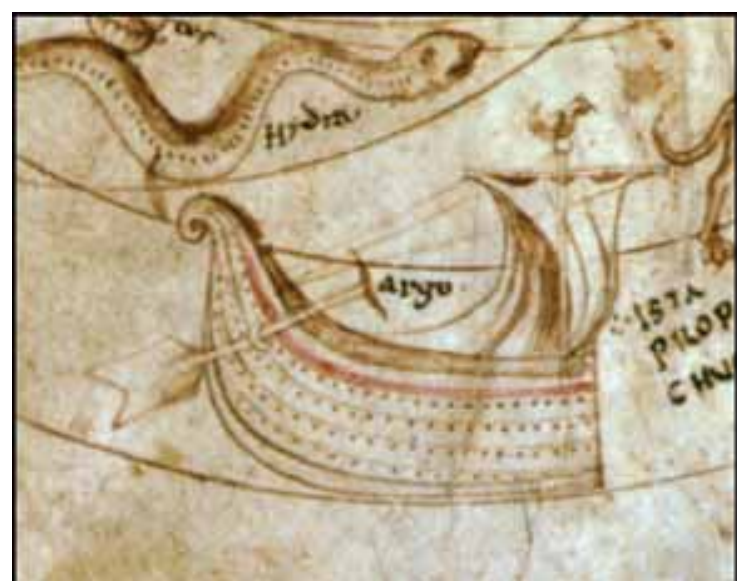

Abb. 12

Das Schiff (Argo) in der Planisphäre des Codex Harleianus 647 (fol. 21v.). 9.-11. Jh. London, British Museum. Fot. British Museum.

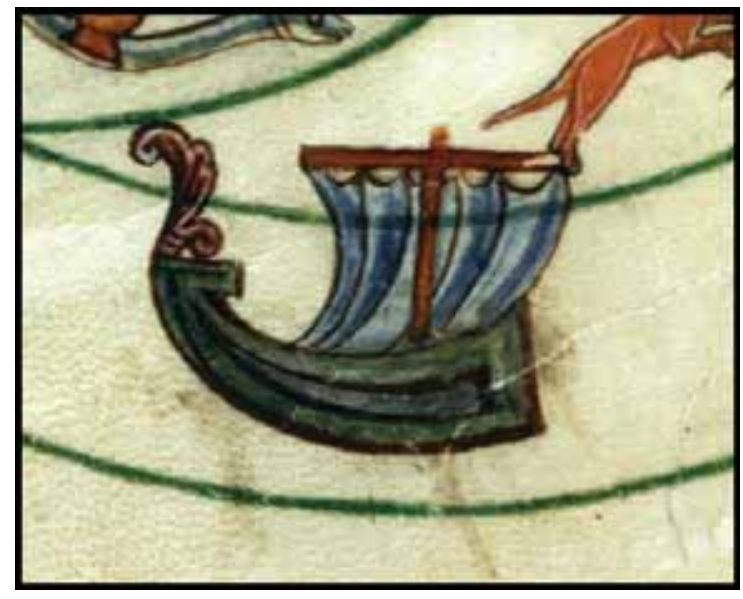

Abb. 13

Das Schiff (Argo) in der Planisphäre des Codex Bernensis 88 (fol.11v.). Vor 1029. Fot. Burgerbibliothek Bern. 


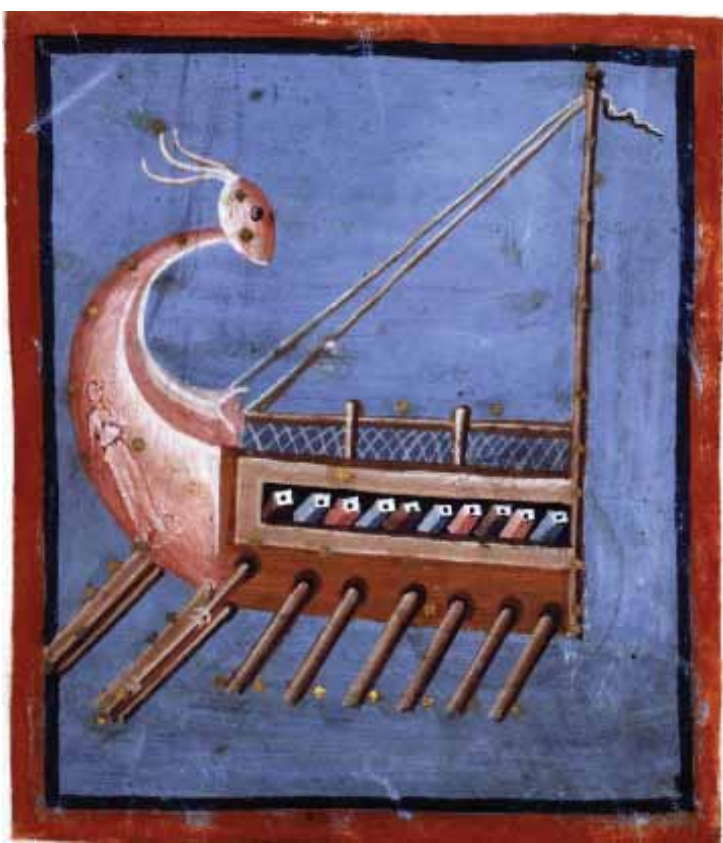

Abb. 14

Das Schiff (Argo) im Codex Vossianus latinus Q 79-64v. Karolingische

Prachthandschrift des Aratos. Um 840.

Leiden, Universiteitsbibliotheek. Phot. Leiden, Universiteitsbibliotheek.

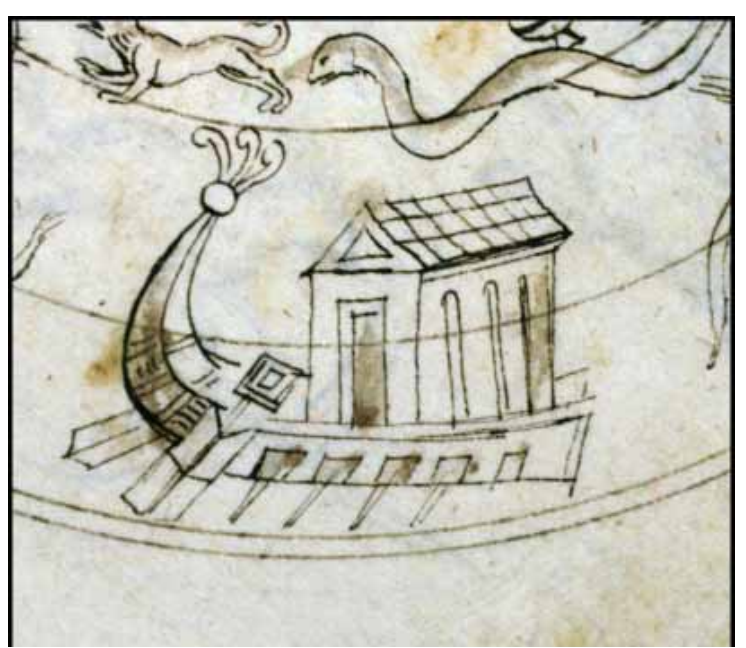

Abb. 15

Das Schiff (Argo) in der Planisphäre des Codex Vaticanus graecus 1087 (fol. 310v). 15. Jh. Rom, Vatikan, Biblioteca Apostolica Vaticana. Fot.

Biblioteca Apostolica Vaticana. hundert. Das Schiff als Ruderschiff des Typs Neumagen zeigt auch schon der ebenfalls aus dem 9. Jh. stammende Codex Vossianus lat. 79 (Abb. 14) ${ }^{25}$, die Überlieferung hielt sich also über lange Zeit.

Die Hyginushandschrift des 9. Jahrhunderts in Dresden, Codex Dresdensis Dc. 183 fol. 13, zeigt ebenfalls das Schiff im Typus des Neumagener Kriegsschiffs, wenn auch vereinfacht ohne Ruder, aber mit den beiden Steuerrudern ${ }^{26}$. Die Darstellung des Einzelbildes Argo im Bernensis 88 (fol. 7r.) folgt dem Vorbild des Codex Vossianus (Abb. 14), in beiden Fällen wird ein leichtes antikes Militärboot dargestellt. Es ist ganz interessant zu sehen, dass im Codex Bernensis 88 das Einzelbild Argo als Kriegsschiff (fol. 7r) von dem Frachtschiff der Planisphäre derselben Handschrift (Abb. 13. fol 11v.) ganz verschieden ist; daran hat man sich offensichtlich nicht gestört.

Die Schiffe des Atlas Farnese (Frachtschiff) und des Globus in Mainz (Militärboot) geben demnach fundamental unterschiedliche Quellen wieder.

Wie lange sich seltene Bildvarianten hielten, zeigt der liegende Widder in Albrecht Dürers Nordhemisphäre von 1515 (Abb. 16) ${ }^{27}$. Wenn man auch seine direkten Quellen nur ein oder indirekt etwa drei Jahrhunderte zurückverfolgen kann $^{28}$, so sind doch die Verbindungen zu den antiken Darstellungen (Abb. 4) anderthalb Jahrtausende zuvor offensichtlich - die lange Dauer solcher Bilder bezeugend.

Es sind uns die antiken Bilder der Helden und Tiere am Himmel so vertraut geworden, dass ein wichtiger Aspekt kaum ins Auge fällt: Die antike Ikonographie der Sternkonstellationen konnte sich über das Mittelalter hinweg gegen jegliche Christianisierung behaupten. Schon in der Spätantike und dann im Laufe des Mittelalters versuchte

25 Thiele 1898: 123f. Fig. 48.

26 ibidem: 43 Fig. 7; Stückelberger 1994: 32 Anm. 17; Obrist 2001: 25 Abb. 16.

27 Benndorf u.a. 1903: 36 Abb. 17; Gundel 1992: 314 Nr. 400.

28 P. Kunitzsch, briefl. 1. 7. 2004. 
man aus christlicher Sicht, die zwölf Tierkreiszeichen in die zwölf Apostel umzubenennen. Den liegenden Widder als apokalyptisches, leidendes Lamm zu interpretieren, gehörte in dieselbe Kategorie $^{29}$. Im 17. Jahrhundert hat man die zwölf Apostel sogar auf ihre Symbole zu reduzieren gesucht, und dafür einen lateinischen Zweizeiler erfunden. Ein Schüler des großen Astronomen Johann Bayer in Augsburg, Julius Schiller, veröffentliche 1627 sein Coelum Stellatum Christianum, einen christlichen Sternenatlas, in dem beispielsweise der Nördliche Kranz (Corona Borealis) als Dornenkrone erscheint.

Dauerhaften Erfolg hatten diese Bestrebungen nicht. Gerade im Dreißigjährigen Krieg, Deutschlands historisch folgenreicher Katastrophe, hatten die Menschen andere Sorgen, als sich um die Umbenennung von Himmelsbildern zu kümmern. Schon im Mittelalter hatten die germanischen Himmelsnamen ${ }^{30}$ die Christianisierung Nordeuropas nicht überlebt. So ist es beim klassischen gräko-römischen Sternenhimmel geblieben.

\section{BIBLIOGRAPHIE}

\begin{abstract}
Abry, J. H. 1993
Hrsg., Les tablettes astrologiques de Grand <Vosges $>$ et l'astrologie en Gaule Romaine. Actes de la Table-Ronde du 18 mars 1992, Université Lyon III, Collection du Centre d'Études Romaines et Gallo-Romaines N.S. 12, Paris: de Boccard.
\end{abstract}

Benndorf, O., Weiss, E. und Rehm, A. 1903 Zur Salzburger Bronzescheibe mit Sternbildern. Jahreshefte des Österreichischen Archäologischen Instituts VI: 32-49.

\section{Bockius, R. 2006}

Die spätrömischen Schiffswracks aus Mainz. Schiffsarchäologisch-technikgeschichtliche Un-

29 Hübner 1983; Hübner 1984: 162.

30 Reuter 1934.

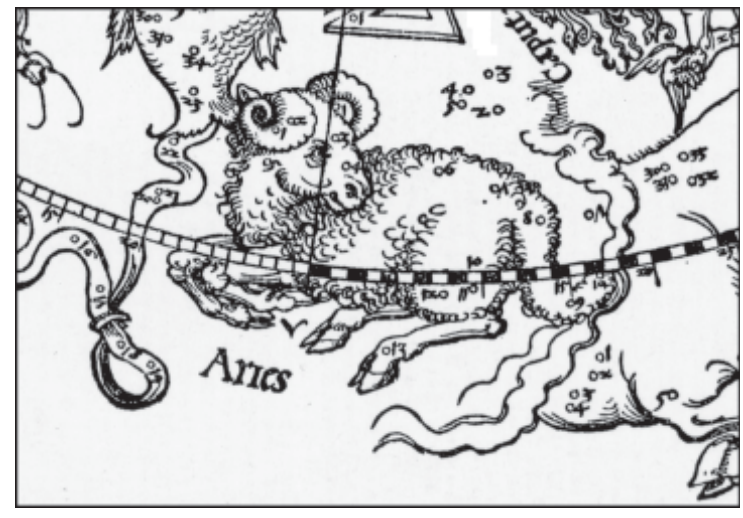

Abb. 16

Albrecht Dürer, Nordhemisphäre, 1515. Liegender Widder (Aries) in der Tradition des hellenistisch-römischen Ägyptens. Wien, Österreichische Nationalbibliothek. Nach Benndorf u.a. 1903, 36 Abb. 17 (Ausschnitt). tersuchung spätantiker Schiffsfunde vom nördlichen Oberrhein, Römisch-Germanisches Zentralmuseum Monographien 67, Mainz: Römisch-Germanisches Zentralmuseum.

\section{Bockius, R. 2007}

Schifffahrt und Schiffbau in der Antike. Archäologie in Deutschland, Stuttgart: Theiss.

\section{Boll, F. 1903}

Sphaera. Neue griechische Texte und Untersuchungen zur Geschichte der Sternbilder, Leipzig: Teubner.

\section{Casson, L. 1994}

Ships and Seafaring in Ancient Times, London: British Museum.

\section{Gundel, H. G. 1992}

Zodiakos. Tierkreisbilder im Altertum. Kosmische Bezüge und Jenseitsvorstellungen im antiken Alltagsleben, Kulturgeschichte der antiken Welt 54, Mainz: Zabern.

\section{Höckmann, O. 1985}

Antike Seefahrt, München: Beck. 


\section{Hübner, W. 1983}

Zodiacus Christianus. Jüdisch-christliche Adaptationen des Tierkreises von der Antike bis zur Gegenwart, Beiträge zur Klassischen Philologie 144, Königstein, Taunus: Hain.

\section{Hübner, W. 1984}

Manilius als Astrologe und Dichter, in: Aufstieg und Niedergang der römischen Welt II 32,1, W. Haase, H. Temporini (Hrsg.), Berlin, New York: de Gruyter, 126-320.

Künzl, E. 2000

Ein römischer Himmelsglobus der mittleren Kaiserzeit. Studien zur römischen Astralikonographie, Jahrbuch des Römisch-Germanischen Zentralmuseums Mainz 47, 2003, Mainz: Römisch-Germanisches Zentralmuseum, 496-594.

\section{Künzl, E. 2005}

Himmelsgloben und Sternkarten. Astronomie und Astrologie in Vorzeit und Altertum, Stuttgart: Theiss.

\section{Lehr, L. 1971}

Beschreibung und Deutung der zwölf Tierkreiszeichen auf den Großbronzen von Alexandria unter der Regierung des Antoninus Pius: Helvetische Münzenzeitung, 3-9. 33-39. 65-67.

Maaß, E. 1902

Salzburger Bronzetafel mit Sternbildern, Jahreshefte des Österreichischen Archäologischen Institus V, 196-197.

\section{Neugebauer, O. und Parker, R. A. 1969}

Egyptian Astronomical Texts, Decans, Planets, Constellations and Zodiacs, London: Lund Humphries.

\section{Obrist, B. 2001}

La représentation carolingienne du zodiaque. A propos du manuscrit de Bâle, Cahiers de civilisation médiévale 44: 3-33.

\section{Reuter, O. S. 1934}

Germanische Himmelskunde: Untersuchungen zur Geschichte des Geistes, München: J. F. Lehmanns.

\section{Saxl, F. 1915a}

Verzeichnis astrologischer und mythologischer illustrierter Handschriften des lateinischen Mittelalters in römischen Bibliotheken, Sitzberichte der Heidelberger Akademie der Wissenschaften, Heidelberg: Winter.

\section{Saxl, F. 1915b}

Verzeichnis astrologischer und mythologischer illustrierter Handschriften des lateinischen Mittelalters II: Die Handschriften der National-Bibliothek in Wien, Sitzberichte der Heidelberger Akademie der Wissenschaften, Heidelberg: Winter.

\section{Saxl, F., Meier, H. und Bober, H. 1953}

Verzeichnis astrologischer und mythologischer illustrierter Handschriften des lateinischen Mittelalters, III in englischen Bibliotheken, London: Warburg Institute.

\section{Schlachter, A. und Gisinger, F. 1927}

Der Globus. Seine Entstehung und Verwendung in der Antike nach den literarischen Quellen und den Darstellungen in der Kunst, Leipzig, Berlin: Teubner.

\section{Stückelberger, A. 1994}

Bild und Wort. Das illustrierte Fachbuch in der antiken Naturwissenschaft, Medizin und Technik, Kulturgeschichte der antiken Welt 62, Mainz: Zabern. 
Thiele, G. 1898

Antike Himmelsbilder. Mit Forschungen zu Hipparchos, Aratos und seinen Fortsetzern und Beiträgen zur Kunstgeschichte des Sternhimmels, Berlin: Weidmannsche Buchhandlung.

von Euw, A. 1987

Aratea. Sternenhimmel in Antike und Mittelalter, A. Legner (Hrsg.), Köln: Schnütgen-Museum.

\section{REZIME}

ARGO KAO RATNIČKI I KAO

TERETNI BROD. ASTRALNA

IKONOGRAFIJA OD ANTIKE DO SREDNJEG VEKA

\section{KLJUČNE REČI: ZVEZDANO NEBO, IKONOGRA-} FIJA, ANTIKA, SREDNJI VEK.

U prošlosti, 48 konstelacija na nebeskom svodu tumačene su kao slike ljudi, životinja ili predmeta. Grci i Rimljani su u ovome pratili uzore preuzete iz Mesopotamije i Egipta, iako su slike sazvežđa formirali na sebi svojstven način. Odlučujući medijum bilo je književno slikarstvo. U izboru motiva se ogledaju život i iskustveni svet ilustratora. Tako je sazvežđe Gemina (Blizanci) predstavljano na dva različita načina, oslanjajući se na dva različita mita. Za sazvežđe broda (Argo), postavljeno daleko na jugu, upotrebljavani su različiti motivi brodova koji su u tom trenutku bili u upotrebi, u jednom slučaju čak izgled sasvim modernog ratničkog broda rimske mornarice. Srednjevekovna izdanja antičkih astronoma se, kada su u pitanju ilustracije, u mnogim elementima oslanjaju na antičke uzore. 